\title{
MRI and contrast-enhanced ultrasound imaging for evaluation of focal irreversible electroporation treatment: results from a phase I-II study in patients undergoing IRE followed by radical prostatectomy
}

\author{
Willemien van den Bos ${ }^{1,7}$ - D. M. de Bruin ${ }^{1,2}$ - A. van Randen ${ }^{3}$ - M. R. W. Engelbrecht ${ }^{3}$ • \\ A. W. Postema ${ }^{1}$ - B. G. Muller ${ }^{1}$ - I. M. Varkarakis ${ }^{4}$ - A. Skolarikos ${ }^{4}$. \\ C. D. Savci-Heijink ${ }^{5}$ R. R. Jurhill ${ }^{5}$ P. J. Zondervan ${ }^{1}$ • M. P. Laguna Pes ${ }^{1}$ • \\ H. Wijkstra ${ }^{1,6}$ - T. M. de Reijke ${ }^{1}$ J. J. M. C. H. de la Rosette ${ }^{1}$
}

Received: 16 July 2015 / Revised: 15 September 2015 / Accepted: 21 September 2015 /Published online: 8 October 2015

(C) The Author(s) 2015. This article is published with open access at Springerlink.com

\begin{abstract}
Objectives Irreversible electroporation (IRE) is an ablative therapy with a low side-effect profile in prostate cancer. The objective was: 1) To compare the volumetric IRE ablation zone on grey-scale transrectal ultrasound (TRUS), contrastenhanced ultrasound (CEUS) and multiparametric MRI (mpMRI) with histopathology findings; 2) To determine a reliable imaging modality to visualize the IRE ablation effects accurately.

Methods A prospective phase I-II study was performed in 16 patients scheduled for radical prostatectomy (RP). IRE of the
\end{abstract}

ClinicalTrials.gov Identifier: NCT01790451

Willemien van den Bos

w.vandenbos@amc.uva.nl

1 Department of Urology, Academic Medical Center, University of Amsterdam, P.O. Box 22660, 1100 DD Amsterdam, The Netherlands

2 Department of Biomedical Engineering \& Physics, Academic Medical Center, University of Amsterdam, P.O. Box 22660, 1100 DD Amsterdam, The Netherlands

3 Department of Radiology, Academic Medical Center, University of Amsterdam, P.O. Box 22660, 1100 DD Amsterdam, The Netherlands

4 2nd Department of Urology, University of Athens, Sismanoglio General Hospital, 1 Sismanogliou Street, 15126 Marousi, Greece

5 Department of Pathology, Academic Medical Center, University of Amsterdam, P.O. Box 22660, 1100 DD Amsterdam, The Netherlands

6 Department of Electrical Engineering, Eindhoven University of Technology, P.O. Box 513, 5600 MB Eindhoven, The Netherlands

7 Academic Medical Center, University of Amsterdam, Meibergdreef 9, 1105 AZ Amsterdam, The Netherlands prostate was performed 4 weeks before RP. Prior to, and 4 weeks after the IRE treatment, imaging was performed by TRUS, CEUS, and mpMRI. 3D-analysis of the ablation volumes on imaging and on H\&E-stained whole-mount sections was performed. The volumes were compared and the correlation was calculated.

Results Evaluation of the imaging demonstrated that with T2weighted MRI, dynamic contrast enhanced (DCE) MRI, and CEUS, effects of IRE are visible. T2MRI and CEUS closely match the volumes on histopathology (Pearson correlation $r=$ 0.88 resp. 0.80). However, IRE is not visible with TRUS.

Conclusions mpMRI and CEUS are appropriate for assessing IRE effects and are the most feasible imaging modalities to visualize IRE ablation zone. The imaging is concordant with results of histopathological examination.

Key points

- mpMRI and contrast-enhanced ultrasound are appropriate imaging modalities for assessing IRE effects

- mpMRI and CEUS are the most feasible imaging modalities to visualize IRE ablation zone

- The imaging is concordant with results of histopathological examination after IRE

- Grey-scale US is insufficient for assessing IRE ablations

Keywords Imaging $\cdot$ Focal therapy $\cdot$ Irreversible electroporation $\cdot$ IRE $\cdot$ Prostate cancer
Abbreviations
Pca Prostate cancer
MRI Magnetic Resonance Imaging
IRE Irreversible electroporation 


$\begin{array}{ll}\text { RP } & \text { Radical prostatectomy } \\ \text { TRUS } & \text { Transrectal ultrasound } \\ \text { CEUS } & \text { Contrast-enhanced ultrasound } \\ \text { mpMRI } & \text { Multiparametric MRI } \\ \text { DCE } & \text { Dynamic contrast enhanced } \\ \text { DWI } & \text { Diffusion-weighted imaging } \\ \text { V } & \text { Volt }\end{array}$

\section{Introduction}

Prostate cancer (Pca) is the most prevalent cancer in males in the Western world and the second most common cause of cancer death. Nevertheless, the majority of the patients will either have their cancer successfully cured or will die with rather than because of prostate cancer [1]. Since the liberal use of prostate-specific antigen tests and Pca screening began, the incidence of Pca diagnosis has increased substantially. Pca is traditionally managed by active surveillance or radical treatments, including radical prostatectomy and radiation therapy [2]. These radical methods often cause concomitant damage to prostate adjacent tissues, resulting in side-effects which include urinary incontinence (9.4-18.3\%), impotence (40$95 \%$ ) and bowel complications (21.9-35.8\%) [3]. Nowadays, the concept of ablative therapy emerges as an approach positioned between expectant management and radical therapy in order to provide effective treatment while minimizing morbidity [4, 5]. In a select group of patients with localized low- and intermediate-risk Pca, ablative therapy might be a more suitable therapeutic option than total gland treatment. The clear purpose is to reduce the toxicity of organ-confined Pca treatments while adequately treating the cancer.

Irreversible electroporation (IRE) is an ablative technology that uses high voltage, low energy direct current, travelling between at least two electrodes. The electric current causes pore formation in the cell membrane that leads to permanent defects that result in cell death [6]. Literature on IRE reports advantages by sparing neighbouring vital structures such as the urethra, blood vessels and nerves [7, 8]. These properties could help to reduce, or even avoid, side effects.

For focal therapy, high quality imaging is of paramount importance for several reasons. The tumour should be identified and extracapsular extension should be excluded. During treatment, the urologist should be confident that the identified tumour area is completely ablated and no significant volume of tumour resides outside the targeted zone. During follow-up, recurrences or residual tumour should be recognized [9]. No literature is available on the role of imaging in follow-up of IRE in prostates. It is unclear what is to expect as image within state-of-the-art imaging modalities like greyscale transrectal ultrasound (TRUS), contrast-enhanced ultrasound (CEUS) or any multiparametric MRI (mpMRI) modality. We hypothesize that 1) TRUS, CEUS and mpMRI are feasible to identify the focal IRE ablation zone in 3D; and 2) ablation zones identified on imaging are comparable to histopathology.

The aim of this study is to identify appropriate methods for the assessment of the IRE treatment zone and to determine which imaging modality (TRUS, CEUS or mpMRI) is feasible to accurately visualize the focal IRE ablation effect in $3 \mathrm{D}$.

\section{Materials and methods}

A prospective study was conducted in 16 patients with confirmed organ-confined prostate cancer who were scheduled for radical prostatectomy (RP). Inclusion and exclusion criteria are listed in Table 1 . The patients underwent a focal IRE procedure approximately one month prior to the surgery. mpMRI [by means of T2-weighted imaging (T2W), dynamic contrast-enhanced imaging (DCE) and diffusion-weighted imaging (DWI)], TRUS, and CEUS imaging were performed prior to the IRE procedure and approximately 4 weeks after the IRE treatment, on the day of admission for the RP. Patient characteristics are presented in Table 2. The study was conducted in two participating centres: AMC University Hospital, Amsterdam, and Sismanoglio University Hospital, Athens. The study was approved by the two individual research ethics committees and registered in the clinicaltrials.gov database (NCT01790451). The trial was executed according to the study protocol previously reported in Van den Bos et al. [10].

\section{IRE}

The IRE procedures were performed using the Nanoknife ${ }^{\circledR}$ IRE system (AngioDynamics Inc, Queensbury, NY) under general anaesthesia and full paralysis. The electrodes were placed transperineally in the prostate under ultrasound guidance (Fig. 1), delivering 90 pulses of $90 \mu$ s duration each with an electric field of on average 1500 Volt (V) per cm distance between the electrodes (voltage-to-distance ratio). The standard setting of $1500 \mathrm{~V} / \mathrm{cm}$ was adapted when the current showed a constant low amperage or high amperage ranging from 1200 to $2100 \mathrm{~V} / \mathrm{cm}$ (Table 3). This was the initial experience of IRE in both participating centres. The number and configuration of the electrodes were varied to assess the effect on the ablation area. The electrodes were placed in the lobe found most positive biopsies. Therefore, exact targeting of the tumour was not pursued.

In the first patient, the IRE procedure was performed using two electrodes. Five patients were treated with three electrodes and ten patients with four electrodes or more. In patient 6 , a pullback of $15 \mathrm{~mm}$ of the electrodes was performed after the first ablation cycle. In patient 8 , the two lateral electrodes were repositioned to the other lobe after one ablation cycle, in order to perform a bilateral ablation. Additional details about the device and procedures are described in BMJ Open [10]. 
Table 1 Inclusion and exclusion criteria

Inclusion criteria
Patients with prostate cancer with indication for radical prostatectomy
Life expectancy $>10$ years
Able to visualize prostate gland adequately on transrectal US imaging
No prostate calcification greater than $5 \mathrm{~mm}$
Ability of subject to stop anticoagulant and anti-platelet therapy for
7 days prior and 7 days post procedure
Exclusion criteria
Bleeding disorders
Active urinary tract infection
History of bladder neck contracture
Inflammatory bowel diseases
Concurrent major debilitating illness
ICD / Pacemaker /Cardiac History
Prior or concurrent malignancy
Biologic therapy for prostate cancer
Chemotherapy for prostate cancer
Hormonal therapy for prostate cancer within 3 months of procedure
Radiotherapy for prostate cancer
Transurethral prostatectomy or urethral stent
Prior major rectal surgery

\section{Greyscale transrectal ultrasound}

Greyscale TRUS scanning was performed in all 16 patients before the IRE procedure and 4 weeks after IRE. Patients were scanned in Amsterdam using a Philips IU22 Ultrasound system with an end-firing transrectal probe (Phillips Healthcare, Bothell, WA, USA) and in Athens using a Falcon 2101 EXCL

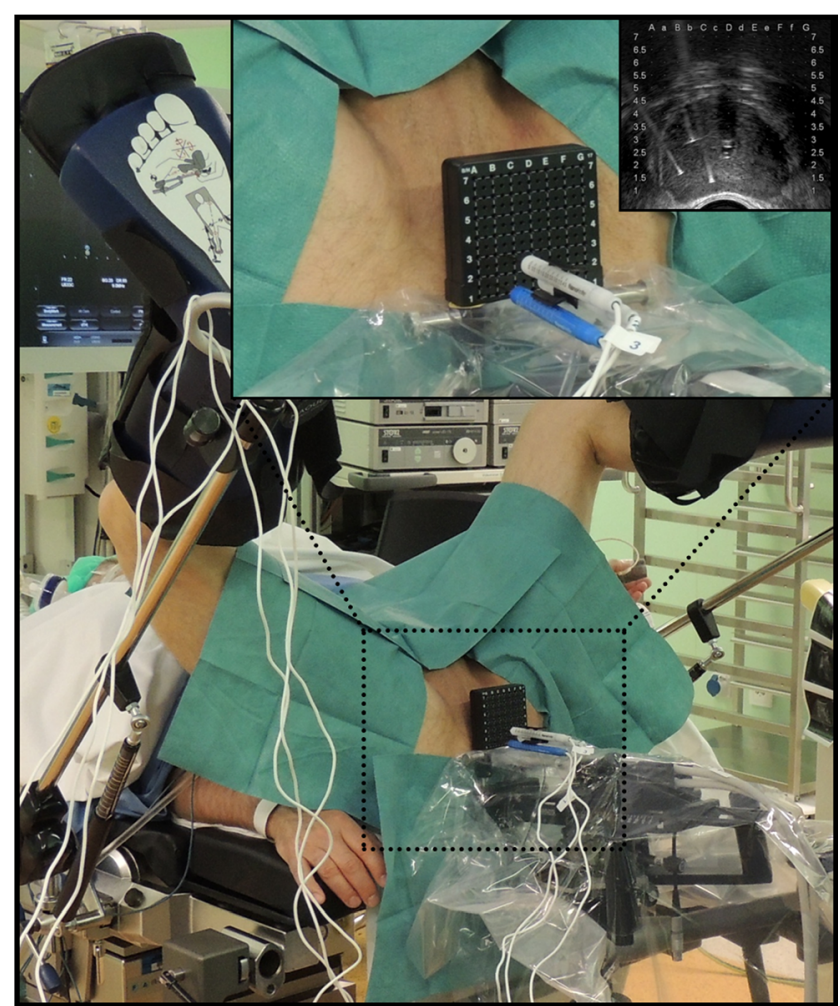

Fig. 1 Patient positioned in high-lithotomy position with three ultrasound-guided transperineally inserted electrodes

(BK Medical, MA, USA) while positioned in the left lateral decubitus position. Greyscale TRUS standard volumetry was performed, followed by three sweeps for later 3D reconstruction, consisting of a transversal sweep from base to apex, a longitudinal sweep from base to apex, and a longitudinal sweep from left to right.

Table 2 Patient characteristics

\begin{tabular}{llllc}
\hline Patient No. & Age & Tumour stage & Gleason score in biopsies & PSA at diagnosis \\
\hline 1 & 63 & T2b & $4+3$ & 13.1 \\
2 & 54 & T2c & $4+3$ & 5.8 \\
3 & 70 & T2b & $3+3$ & 5 \\
4 & 65 & T2c & $3+3$ & 9.6 \\
5 & 48 & T2a & $4+4$ & 4.4 \\
6 & 69 & T2a & $3+3$ & 13 \\
7 & 44 & T2b & $3+3$ & 3.6 \\
8 & 69 & T2c & $3+3$ & 5.7 \\
9 & 56 & T2c & $3+3$ & 8.7 \\
10 & 75 & T1c & $4+3$ & 15.5 \\
11 & 50 & T1c & $3+4$ & 5.3 \\
12 & 73 & T2a & $3+4$ & 25 \\
13 & 49 & T2c & $3+4$ & 7 \\
14 & 54 & T1c & $3+3$ & 8 \\
15 & 60 & T1c & $3+3$ & 5.8 \\
16 & 63 & T2c & $4+4$ & 10.1 \\
\hline
\end{tabular}


Table 3 Procedure specifications per patient

\begin{tabular}{llll}
\hline Patient No. & Volume prostate $(\mathrm{mL})$ & Number of electrodes & Voltage-to-distance ratio (V/cm) \\
\hline 1 & 50 & 2 & 1500 \\
2 & 30 & 3 & $1500-1700$ \\
3 & 57 & 4 & 1500 \\
4 & 60 & 4 & $1200-1500$ \\
5 & 19 & 3 & $1500-1800$ \\
6 & 42 & $4+$ pullback & $1350-1500$ \\
7 & 21 & 3 & $1500-1650$ \\
8 & 40 & 6 & $1350-1500$ \\
9 & 50 & 4 & $1500-1800$ \\
10 & 37 & 4 & 1500 \\
11 & 25 & 3 & $1500-1800$ \\
12 & 50 & 4 & $1500-1650$ \\
13 & 28 & 4 & $1500-1800$ \\
14 & 36 & 4 & $1500-1950$ \\
15 & 31 & 3 & $1500-2100$ \\
16 & 48 & 3 & 1500 \\
\hline
\end{tabular}

\section{Contrast-enhanced ultrasound}

Subsequently, CEUS recordings were acquired before and 4 weeks after IRE in the 12 patients treated in Amsterdam. CEUS imaging was performed with the same Philips IU22 machine using the protocol described by Kuenen et al. [11] with an ultrasound contrast agent that consists of gas-filled microbubbles, which are stabilised by a protein or lipid shell (Sonovue $^{\circledR}$, Bracco, Milan, Italy). The prostate was scanned in four planes: base, base-mid, mid-apex, and apex. For each plane, recordings were completed after a bolus injection $2.4 \mathrm{~mL}$ of UCA followed by a $5 \mathrm{~mL}$ saline flush. Subsequently, transversal sweeps from base to apex were performed to allow calculation of the dimensions of the contrast deficits after IRE.

\section{Multiparametric MRI}

Two mpMRI studies were performed per patient. The first was acquired prior to the IRE procedure and the second was performed, 4 weeks after the IRE treatment and 1 day prior to RP. The mpMRIs were made according to the ESUR guidelines [12]. All patients included in the AMC Amsterdam ( $n=12$ patients) underwent mpMRI performed on a 1.5 Tesla AVANTO ${ }^{\circledR}$ MRI scanner (Siemens Healthcare, Erlangen, Germany) using an integrated endorectal-pelvic phased-array coil (Medrad, Warrendale, USA).[13] The mpMRI protocol in Athens ( $n=4$ patients) was performed on a 3.0 tesla MAGNETOM Trio (Siemens, Healthcare, Erlangen, Germany) with a pelvic phased-array coil. In the $\mathrm{AMC}$, first, $\mathrm{T} 2$ weighted turbospin echo sequences were performed in axial, coronal, and sagittal planes, covering the prostate and seminal vesicles. Second, a singleshot-echo-planar imaging sequence with diffusion module and fat suppression pulse was implemented. ADC maps were automatically calculated by the scanner software, using all three b-values. Next, dynamic gadolinium enhanced MRI was performed using fast gradient echo sequences. Seven slices covering the prostate were acquired with a temporal resolution of seven slices per 2.1 seconds before and 90 seconds after intravenous administration of $0.1 \mathrm{mmol}$ of gadopentetate dimeglumine per $\mathrm{kg}$ of body weight Gadolinium DTPA (Gadovist). To suppress bowel peristalsis, intravenous or intramuscular administration of $20 \mathrm{mg}$ Buscopan ${ }^{\circledR}$ was given. In Athens at 3T, first T2-weighted turbospin echo sequences were performed in transverse, coronal and sagittal directions. Next, single-shot-echo-planar imaging sequences with diffusion module and fat suppression pulse were implemented. ADC maps were automatically calculated by the scanner software using two b-values. Finally, dynamic contrast-enhanced MRI was performed using fast gradient echo sequences with a similar temporal resolution as in the AMC.

\section{D assessment of imaging}

The pre- and post-treatment images of the prostates were examined in consensus by two uro-radiologists of the AMC, blinded to pathology results. Cross-sectional images from TRUS, CEUS, and mpMRI were visually inspected for lesion boundaries and contrast differences. The ablation zones were volumetrically evaluated in 
Fig. 2 Imaging modalities preIRE (left) and 4 weeks post-IRE (right): $a$. Grey-scale ultrasound; cannot image the ablation zone. $b$. CEUS; shows a sharp-demarcated dark area presenting non-perfused tissue. $c$. DCE-MRI shows a sharp-delineated non-enhancing lesion. $d$. T2-weighted MRI shows hypo intense lesion

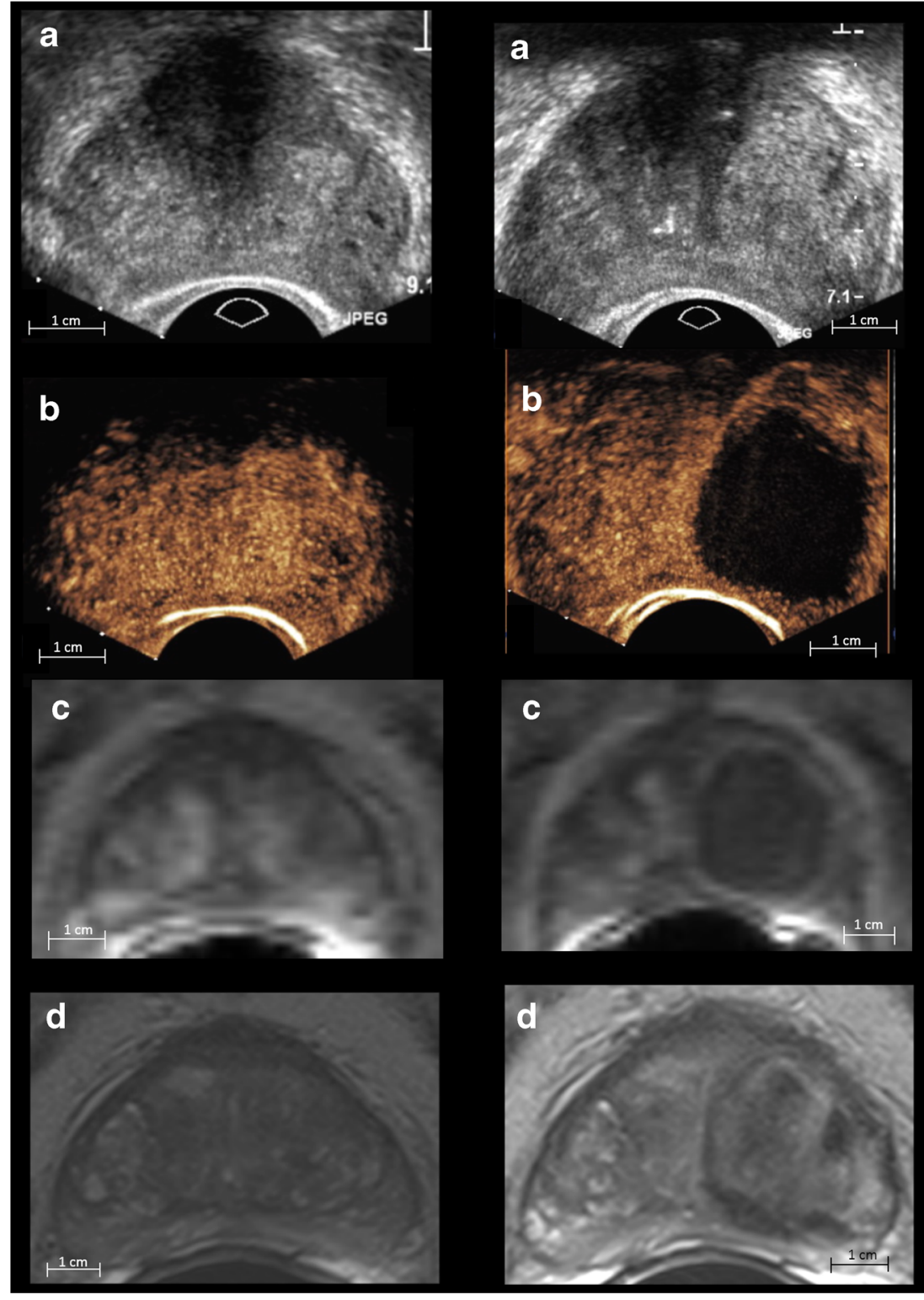

CEUS and T2 MRI data. For MRI, the ablation zones were manually segmented frame by frame, using a delineation tool using Amira ${ }^{\circledR}$. For CEUS, the ablation zones were automatically segmented in $3 \mathrm{D}$ using averaging, window levelling and wand tracing in ImageJ/ FIJI [14], or manually, frame by frame using a delineation tool. The precisely outlined areas were segmented and aligned to create a $3 \mathrm{D}$ reconstruction of the ablation zones, and the volumes were determined using AMIRA shape-analysis package software (Version 5.5; FEI Visualization Sciences Group, Mérignac Cédex, France).

\section{Histopathology}

To ensure consistency of histopathological analysis, examination of the prostate specimens from both centres was performed at the pathology department of the AMC. The serial sections of 3-5 $\mathrm{mm}$ were cut perpendicular to the rectum according to the axial MRI plane, followed by a surface cut of each slice, and these were inspected macroscopically and documented by photography. Whole-mount slices from apex to base were embedded in paraffin; 4- $\mu$ m-thick sections were cut and examined with H\&E staining. The boundaries of the ablation zone were determined manually using light microscopy, and marked on the slides. The outlined slides were scanned with an IntelliSite Ultra-Fast Scanner (Philips, Best, the Netherlands), and the volumes of tissue alterations were defined by segmentation as indicated by the pathologist, followed by volumetric shape analysis in AMIRA. The acquired volumes were adjusted for shrinkage during fixation using the pre-fixation prostate dimensions divided by the post-fixation volume. 


\section{Data analysis}

All extracted volumes of CEUS, T2 MRI and PA were directly compared and visualised using an $\mathrm{x} / \mathrm{y}$ scatterplot. The scatter data was linearly fitted, from which a Pearson correlation value (goodness of fit, $r$ ) and slope were calculated as measures of correlation. All the images were matched with the PA volumes. To calculate the error per 3D reconstruction for CEUS, T2 MRI and PA, the lesion volume was divided by the number of slices from which the 3D data set was constructed.

\section{Results}

\section{Procedural results}

No serious adverse event was observed during the procedure or hospital stay. All patients, except for one due to social reasons, were discharged from the hospital 1 day after the IRE treatment.

\section{Qualitative evaluation of imaging}

Qualitative evaluations of the imaging modalities showed that grey-scale TRUS inadequately visualized IRE ablation effects in all 16 cases. All post-IRE CEUS provided a clear, dynamic, homogeneous, non-perfused image of the ablation zone in all patients, except for the first treated patient, in which no non-perfused area could be detected. In nine out of 12 scans, the data set was sufficient to reconstruct the ablation zone in $3 \mathrm{D}$.

On T1-dynamic scans, the IRE effects are identifiable as areas of non-contrast enhancement. On T2W images, 15 patients showed heterogeneous signal intensity and nine patients showed marked hypo-intense margins suggestive of fibrosis. The mpMRI of the first patient did not show a clear ablation zone. Three of the patients had a slightly higher intralesional T2-signal intensity, possibly because of oedema. Twelve patients had high signal intensity focal intralesional areas due to haemorrhage on the pre-contrast T1-weighted images. TRUS and CEUS images were simultaneously acquired and are therefore automatically matched. mpMRI images were visually matched to the CEUS data (Fig. 2). Three-dimensional reconstructions are shown in Fig. 3.

\section{Qualitative evaluation of histopathology}

Macroscopic assessment showed, in 15 cases, lesions in the treated zone with a central white necrotic zone surrounded by an outer, dark red haemorrhagic zone (Fig. 4). Microscopic assessment showed, in the same 15 cases, fibrotic and necrotic areas with mild inflammation with sharp demarcations. The prostate specimen of the first treated patient contained solely fibrotic tissue in a bilateral pattern without any necrotic component, not showing any relation to the unilateral electrode configuration. This suggests that the fibrosis was pre-existent and
Fig. 3 1. PA whole-mount slide with delineated ablation zone (dotted line) and 3D reconstruction of ablation zone. 2 . T2-MRI with 3D-recontruction of prostate and ablation zone. 3 . CEUS of prostate with 3Drecontruction of ablation zone

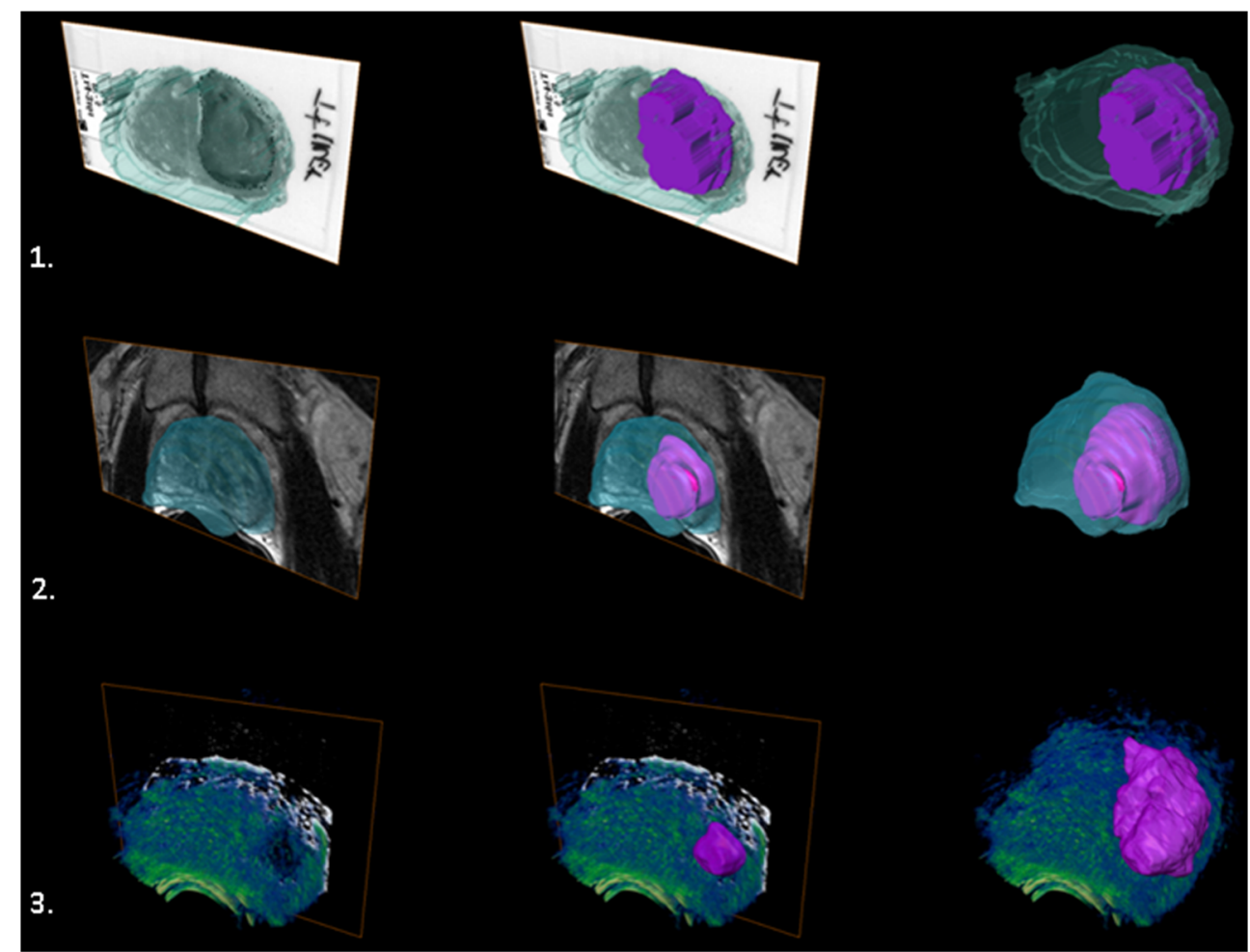


Fig. 4 Macroscopic fixed 4-mmthick prostate slice and wholemount PA coupe with H\&E staining

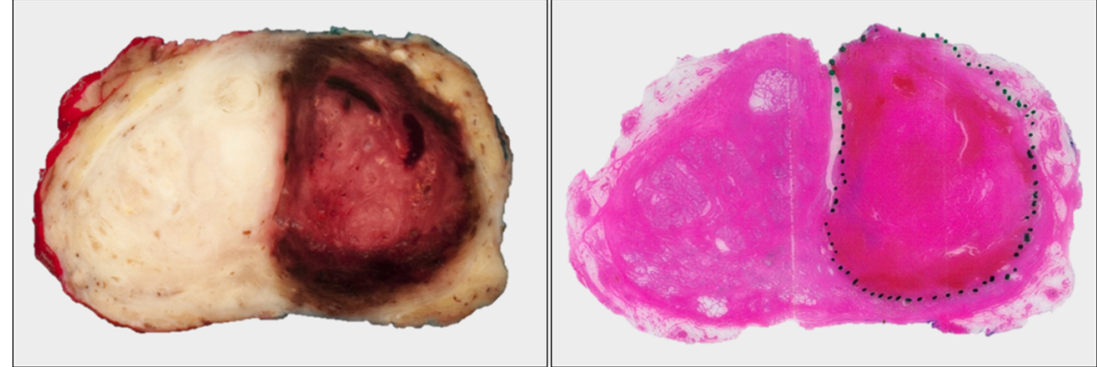

not caused by IRE, and therefore it was excluded from the analysis.

\section{Quantitative evaluation}

\section{mpMRI-PA correlation}

Comparison of the ablation zones volumes traced on the postIRE T2-weighted MR images with the ablated volume traced on H\&E stained PA images revealed that MRI-measured volumes were 1.16 times the PA measurements. The Pearson correlation index between the two sets of measurements was $r=0.88$ with a slope of 0.75 (Fig. $5 \mathrm{~A}$ ).

\section{CEUS-PA correlation}

Ablation zone volumes traced on the post-IRE CEUS images compared with volumes traced on H\&E PA images showed that, on average, CEUS volumes were 1.57 times greater than PA measurements. The Pearson correlation index between these sets of measurements was $r=0.80$ with a slope of 0.82

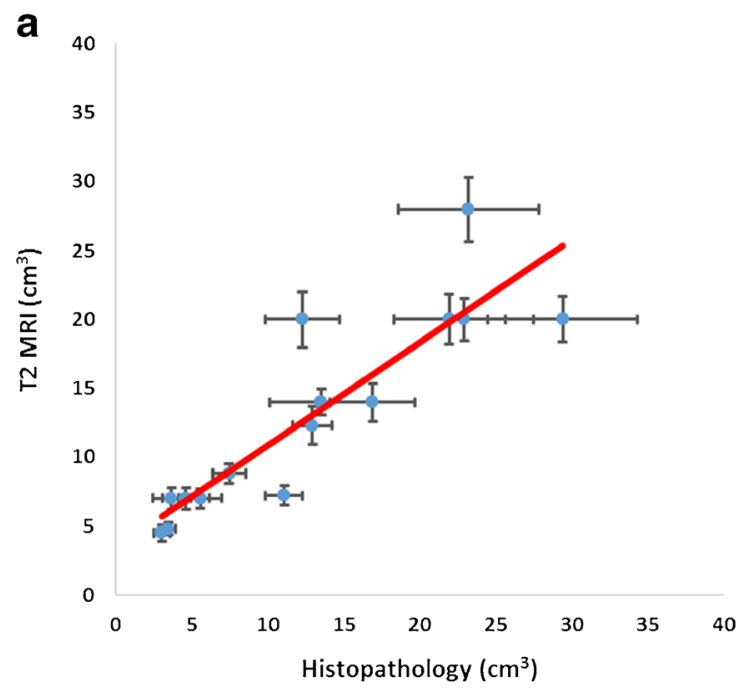

Fig. 5 Correlation of ablation volumes T2-weighted MRI and H\&E pathology $(A)$. The Pearson correlation index between the two sets of measurements was $r=0.88$ with a slope of 0.75 . Correlation of ablation
(Fig. 5B). The errors on CEUS values were too small to display in the graph.

\section{Discussion}

This is the first study assessing three different imaging modalities pre- and post-IRE treatment for prostate cancer. This study demonstrated that either mpMRI or CEUS can be used to accurately and reliably follow the area treated with IRE. Lindner et al. published a similar designed case-study with focal laser ablation [15]. Comparison of the ablated volume traced on H\&E stained PA with the volumes traced on MRI revealed on average that MRI-measured volumes were 1.4 times PA measurements with a Pearson correlation index of $r=0.79$. Grampsas et al. published a comparable trial with cryoablation, concluding that TRUS overestimates the area of prostatic tissue destroyed by the ablation, and this challenges the assumption that the entire prostate is lethally frozen when its boundaries are included within the hypoechoic ice ball observed on TRUS [16]. Several studies have been performed using high-intensity focused ultrasound (HIFU)

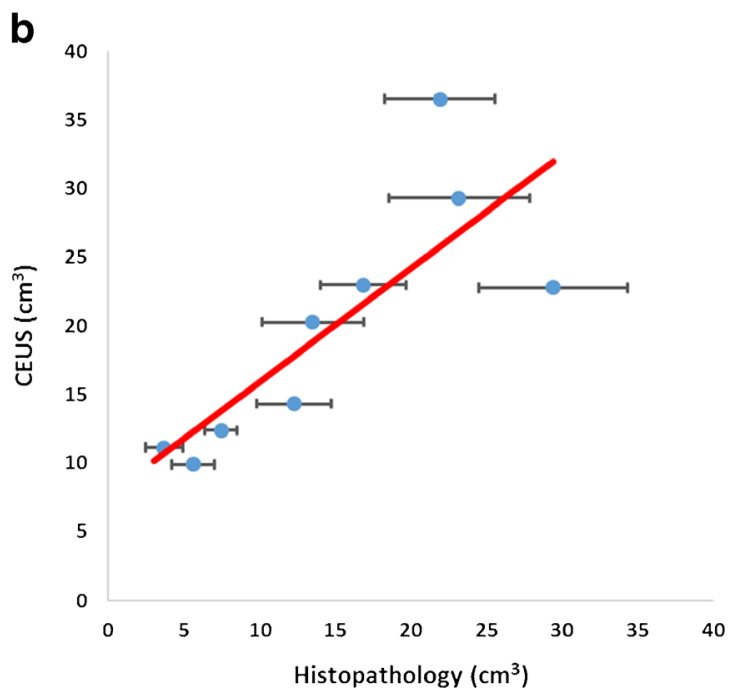

volumes CEUS and H\&E pathology $(B)$. The Pearson correlation index between these sets of measurements is $r=0.80$ with a slope of 0.82 . The errors on CEUS values are too small to display in the graph 
followed by RP. Madersbacher et al. demonstrated that HIFU induced sharply delineated intraprostatic coagulative necrosis within the target area, whereas alterations of periprostatic structures were never observed [17]. The histopathological effects of IRE in correlation with treatment planning and probe placement, in addition to effects to the prostate capsule, urethra and neurovascular bundle, were recently described by Van den Bos et al. [18]. Beerlage et al. showed PA reports of radical prostatectomy specimens following HIFU treatment with marked and complete necrosis in the treated area [19, 20]. None of these studies include post-treatment imaging assessing the ablation effects during follow-up.

A limitation of the present study is the low number of CEUS data sets available for analysis. Because CEUS could only be obtained in Amsterdam, CEUS analysis was done using the data of 12 patients. In nine patients, 3D reconstructions could be made using the CEUS data. In the remaining four scans, the transversal sweep was insufficient for a reliable $3 \mathrm{D}$ analysis. Wiggerman et al. performed CEUS immediately after and 20 minutes after IRE of hepatic tumours and concluded that CEUS allowed good prediction of ablation result following IRE [21]. Furthermore, at this early phase of research, the IRE treatments were performed without curative intent. So within the trial, the efficacy for treatment of prostate cancer could not yet be assessed. Another limitation is that the fixated prostates were cut by hand, possibly leading to reduction of the correlation between the imaging and PA.

In the analysis of the PA, the prostate specimen of the first patient (treated with a two-electrode configuration) showed confluent fibrotic tissue without a necrotic component, in contrast to all other specimens that contained a necrotic focus. Additionally, the IRE treatment was performed unilaterally, yet the fibrosis was present in both lobes and was therefore considered as pre-existent, as associated with chronic inflammation and aging. Together with the additional absence of infield necrotic tissue, the patient was excluded from further analysis. However, Neal et al. [22] also performed IRE treatments using only two electrodes in prostate cancer patients $(n=2)$ that did show PA ablation zones of $1.14 \mathrm{~cm}^{3}$ and $2.46 \mathrm{~cm}^{3}$. This may indicate that the incomplete ablation was not due to the low number of used electrodes, and the cause of the required exclusion of this patient remains unknown.

The close matches of T2MRI, DCE-MRI and CEUS with PA confirm the accuracy of these imaging modalities in rendering the ablation zone. The latter two modalities are based on tissue perfusion and both modalities clearly show a loss of enhancement that represents devascularisation and thus tissue destruction. Since the PA showed mainly end-stage fibrotic tissue, the destruction of tissue 4 weeks post-IRE is reasonably representing the long-term effects. So, ablation effects may be reasonably measurable by these imaging modalities.

\section{Conclusion}

T2MRI, and CEUS are feasible imaging modalities to visualize the IRE ablation effects in 3D with a strong Pearson's correlation of $r=0.88$ and $r=0.80$, respectively, compared with histopathology. Grey-scale US is insufficient for assessing IRE ablation volumes. mpMRI and CEUS should be used in future research for the evaluation of IRE effects of ablation in focal therapy for prostate cancer.

Acknowledgements The scientific guarantor of this publication is J.J.M.C.H. de la Rosette. The authors of this manuscript declare relationships with the following companies: J.J.M.C.H. de la Rosette is a paid consultant for Angiodynamics. All other authors of this manuscript declare no relationships with any companies, whose products or services may be related to the subject matter of the article. This study has received funding by Endourological Society and the Cure for Cancer Foundation. One of the authors has significant statistical expertise. Institutional Review Board approval was obtained. Written informed consent was obtained from all subjects (patients) in this study. Methodology: prospective, phase I-II pilot study, multicentre study.

Open Access This article is distributed under the terms of the Creative Commons Attribution-NonCommercial 4.0 International License (http://creativecommons.org/licenses/by-nc/4.0/), which permits any noncommercial use, distribution, and reproduction in any medium, provided you give appropriate credit to the original author(s) and the source, provide a link to the Creative Commons license, and indicate if changes were made.

\section{References}

1. Siegel R, Ma J, Zou Z, Jemal A (2014) Cancer statistics, 2014. CA Cancer J Clin 64:9-29

2. Heidenreich A, Bastian PJ, Bellmunt J et al (2014) EAU guidelines on prostate cancer. part 1: screening, diagnosis, and local treatment with curative intent-update 2013. Eur Urol 65:124-37

3. Resnick MJ, Koyama T, Fan K-H et al (2013) Long-term functional outcomes after treatment for localized prostate cancer. N Engl J Med 368:436-45

4. Valerio M, Ahmed HU, Emberton M et al (2013) The role of focal therapy in the management of localised prostate cancer: a systematic review. Eur Urol 44:1-20

5. Barret E, Ahallal Y, Sanchez-Salas R, et al (2012) Morbidity of focal therapy in the treatment of localized prostate cancer. Eur Urol 1-5. doi: 10.1016/j.eururo.2012.11.057

6. Lee EW, Wong D, Prikhodko SV et al (2012) Electron microscopic demonstration and evaluation of irreversible electroporationinduced nanopores on hepatocyte membranes. J Vasc Interv Radiol 23:107-13

7. Onik G, Rubinsky B (2010) First patient experience focal therapy of prostate cancer. irreversible electroporation. Springer, Berlin, pp 235-247

8. Li W, Fan Q, Ji Z et al (2011) The effects of irreversible electroporation (IRE) on nerves. PLoS One 6, e18831. doi:10.1371/journal. pone. 0018831

9. Muller BG, van den Bos W, Pinto PA, de la Rosette JJ (2014) Imaging modalities in focal therapy: patient selection, treatment guidance, and follow-up. Curr Opin Urol 24:218-24

10. Van den Bos W, de Bruin DM, Muller BG et al (2014) The safety and efficacy of irreversible electroporation for the ablation of 
prostate cancer: a multicentre prospective human in vivo pilot study protocol. BMJ Open 4:e06382-e006382

11. Kuenen MPJ, Saidov TA, Wijkstra H, Mischi M (2013) Contrastultrasound dispersion imaging for prostate cancer localization by improved spatiotemporal similarity analysis. Ultrasound Med Biol 39:1631-41

12. Barentsz JO, Richenberg J, Clements R et al (2012) ESUR prostate MR guidelines 2012. Eur Radiol 22:746-57

13. Muller BG, van den Bos W, Brausi M et al (2014) Role of multiparametric magnetic resonance imaging (MRI) in focal therapy for prostate cancer: a Delphi consensus project. BJU Int 114: 698-707

14. Schindelin J, Arganda-carreras I, Frise E, et al. (2013) Fiji - an Open Source platform for biological image analysis. doi: 10.1038/nmeth. 2019.Fiji

15. Lindner U, Weersink RA, Haider MA et al (2009) Image guided photothermal focal therapy for localized prostate cancer: phase I trial. J Urol 182:1371-7

16. Grampsas SA, Miller GJ, David Crawford E (1995) Salvage radical prostatectomy after failed transperineal cryotherapy: histologic findings from prostate whole-mount specimens correlated with intraoperative transrectal ultrasound images. Urology 45:936-941
17. Madersbacher S, Pedevilla M, Vingers L et al (1995) Effect of high-intensity focused ultrasound on human prostate cancer in vivo effect of high-intensity focused ultrasound on human prostate cancer in vivo. Cancer Res 55:3346-3351

18. Van den Bos W, de Bruin DM, Jurhill RR et al (2015) The correlation between the electrode configuration and histopathology of irreversible electroporation ablations in prostate cancer patients. World J Urol. doi:10.1007/s00345-015-1661-x

19. Chopra R, Colquhoun A, Burtnyk M et al (2012) MR imagingcontrolled transurethral ultrasound therapy for conformal treatment of prostate tissue: initial feasibility in humans. Radiology 265:30313

20. Beerlage HP, Van Leenders GJ, Oosterhof GO et al (1999) Highintensity focused ultrasound (HIFU) followed after one to two weeks by radical retropubic prostatectomy: results of a prospective study. Prostate 39:41-46

21. Wiggermann P, Zeman F, Niessen C et al (2012) Percutaneous irreversible electroporation (IRE) of hepatic malignant tumours: contrast-enhanced ultrasound (CEUS) findings. Clin Hemorheol Microcirc 52:417-27

22. Neal RE, Millar JL, Kavnoudias H et al (2014) In vivo characterization and numerical simulation of prostate properties for nonthermal irreversible electroporation ablation. Prostate 468:458-468 